\title{
Fecal calprotectin levels are higher in rural than in urban Chinese infants and negatively associated with growth
}

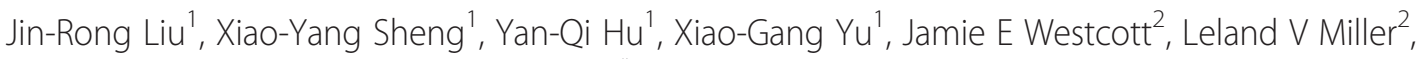
Nancy F Krebs ${ }^{2}$ and K Michael Hambidge ${ }^{2^{*}}$

\begin{abstract}
Background: Fecal calprotectin (FC) is an established simple biomarker of gut inflammation. To examine a possible relationship between linear growth and gut inflammation, we compared fecal calprotectin levels in 6 month old infants from poor rural vs affluent urban families.

Methods: The project was a cross-sectional comparison of FC from rural and urban populations in China. The relationship between length-for-age Z-score (LAZ) and FC concentrations were also compared. Single fecal samples were assayed for FC using EK-CAL ELISA kits.

Results: The age of subjects for both locations was $6.1 \pm 0.2 \mathrm{mo}$; all were apparently healthy. The mean \pm SD of the LAZ for the rural and urban infants were $-0.6 \pm 0.9$ and $0.4 \pm 0.9$, respectively. FC had a non-normal distribution. The median FC of 420.9 and $140.1 \mu \mathrm{g} / \mathrm{g}$ for rural and urban infants, respectively, were significantly different $(P<0.0001)$. For the rural group, linear regression analysis showed that an increase in $\mathrm{FC}$ of $100 \mathrm{\mu g} / \mathrm{g}$ was associated with a decrease of 0.06 in LAZ.
\end{abstract}

Conclusion: FC levels were significantly elevated in the rural infants and high concentrations accounted for approximately one-third of the low LAZ scores of these infants.

Keywords: Fecal calprotectin, Infants, Children, Gut inflammation, Growth

\section{Background}

The unhygienic and unsanitary environment typical of so many children in poor communities worldwide has been incriminated as a factor of note in the early onset of impaired linear growth in infants in these communities [1]. However, quantitative information remains limited on the role of unhygienic, unsanitary, poor environments per se on intestinal inflammation and associated linear growth failure [2-5]. Lunn and Campbell, et al. [2] proposed that frequent gastrointestinal infections, arising from unhygienic and unsanitary environments, impair small intestinal mucosal function, and explained up to $64 \%$ of the observed height and weight faltering of Gambian infants. Recently,

\footnotetext{
* Correspondence: Michael.Hambidge@ucdenver.edu

University of Colorado School of Medicine, Department of Pediatrics, Section of Nutrition, Box C225, Research Complex II, 12700 East 19th Avenue, Aurora, CO 80045, USA

Full list of author information is available at the end of the article
}

works in Bangladesh and Nepal further confirmed the association of gut damage and growth faltering [6,7].

Calprotectin is a calcium and zinc binding protein in the $\mathrm{S} 100$ family with a total molecular mass of $36.5 \mathrm{kD}$ [8-10]. It is abundant in neutrophilic granulocytes, monocytes and macrophages, accounting for $60 \%$ of all cytosolic protein in neutrophils. Neutrophils comprise part of the intestinal innate immune system and pass through the intestinal wall resulting in increase in secretion of calprotectin which is excreted in the feces intact. Fecal calprotectin (FC) is an established biomarker of intestinal inflammation [11-14]. Most recently, fecal calprotectin levels have also been found to be associated with persistent Giardia and microscopic duodenal inflammation [15]. Only a gram or less of sample is required with improved assays and the FC is remarkably resilient to degradation for more than a week at room temperature [16]. The application of FC assays provides

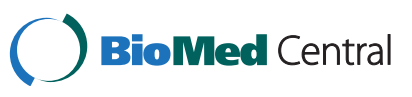


a promising opportunity for comparison of the occurrence and severity of intestinal inflammation between communities exposed to different environments. We hypothesized that FC levels in mid-infancy are higher in a rural population than in a middle income urban population, and that FC levels are negatively associated with the rural infants' growth.

\section{Methods}

\section{Study design}

The project was a cross-sectional comparison of fecal calprotectin concentrations in infants at the age of 6 mo in two Chinese populations: one poor rural, the other upper-middle income urban. We also examined the relationship between length-for-age Z-scores (LAZ) and fecal calprotectin levels.

\section{Subjects}

The subjects were infants aged $6 \pm 1$ mo. One-hundredand-five infants were located in the relatively poor rural county of Xichou located in southwest China. All were apparently healthy with no evidence of acute or chronic disease or congenital malformations. This study resulted from a hypothesis that developed late in the course of 6-mo baseline data collection for the primary study, and rural samples were limited to all infants - no refusals or exclusions and none with recent history of diarrhea who had not already had their baseline visit. Hence sample size was dictated by practical limitations rather than by power testing. The primary study was a complementary feeding study commencing after these baseline studies (Sheng et at, unpublished). The rural subjects were from 60 rural villages in Xichou county. The sample size for the urban infants was also dictated by practical considerations. For a limited time period all infants seen at Kongjiang Community Health Center, Shanghai, for a routine health check at age 6 months were invited to participate with $100 \%$ acceptance. These were healthy term singleton infants from upper-middle class families delivered between 37-42 wk gestation with birth weights of 2,500-4000 g. Infants with history of diarrhea or respiratory tract infection within previous 2 weeks were excluded.

Written informed consent was obtained from the mother of each infant. The research protocol was approved by Shanghai Jiao Tong University School of Medicine Xinhua Hospital Institute Review Board and the Colorado Multiple Institutional Review Board.

\section{Anthropometric measurements}

Infant anthropometric measurements were undertaken in duplicate by a trained member of the research team. Lengths were measured following standard techniques using a portable seca infantometer (seca corp, Hanover,
MD, USA, $0.1 \mathrm{~cm}$ precision) and seca electronic scale (5 g precision). If the two length measurements differed by $>0.4 \mathrm{~cm}$, a third measurement was taken. The mean of the two closest length measurements were recorded.

\section{Fecal collection, transport and storage}

A plastic container was distributed to the mothers who were instructed on collection of a stool sample and to take precautions against contamination with soil or dirt. The stool sample was collected on the same day or a day before the study visit. Samples were stored at $-20^{\circ} \mathrm{C}$. The samples from Yunnan rural infants were transferred frozen to Shanghai.

\section{Calprotectin assays}

The fecal samples were analyzed within three months of collection. FC levels were measured using an EK-CAL ELISA kit (Bühlmann Laboratories AG, Switzerland), following the manufacturer's instructions. The frozen samples were defrosted at room temperature. The empty polypropylene tube together with the inoculation loop was labeled and weighed. Approximately 50 100 mg of the fecal sample were transferred into a pre-weighed tube by means of an inoculation loop. The net amount of sample was weighed then broken off the inoculation loop leaving the lower part of the loop in the tube. Extraction buffer $(49 \times$ net weight) was added to the tube and the tube was closed before sample homogenization on a multi-tube vortexer with vigorous shaking for $30 \mathrm{~min}$. The homogenate was transferred into a $2 \mathrm{~mL}$ polypropylene tube and centrifuged in a microcentrifuge for $5 \mathrm{~min}$ at $3000 \times \mathrm{g}$. The supernatant was transferred into a fresh, labeled tube for the ELISA procedure. The lower range procedure (working range $10 \sim 600 \mu \mathrm{g} / \mathrm{g}$ ) was chosen to perform the ELISA assay in the first two batches which included 98 samples. The fecal extracts were diluted 1:50 with incubation buffer and the absorbance was read at $450 \mathrm{~nm}$ in a microtiter plate reader. Unexpectedly, 29 samples, all but one from the rural infants, read higher than the top calibration. Because no sample material was available for further analysis, these samples could only be quantified as having FC levels $>600 \mu \mathrm{g} / \mathrm{g}$. Subsequently, the remaining extract was stored and if analytical results exceeded the top calibrator, second fecal extracts were further diluted 1:6 with incubation buffer and assayed again.

\section{Statistical analyses}

(LAZ), weight-for-age (WAZ), and weight-for-length (WLZ) Z-scores were calculated with Anthro (version 3.1) using World Health Organization Child Growth standards. Comparison tests of LAZ, WAZ and WLZ were performed using t-tests. Because FC data were found to have a non-normal distribution, comparison 
tests of these data were conducted using the nonparametric Mann-Whitney Test. The non-parametric test also accommodated the presence of the 29 data having unknown fecal calprotectin values $>600 \mu \mathrm{g} / \mathrm{g}$. In cases where the rankings of the unknown data affected the $P$-values of tests, hypothetical FC values that produced the extreme ranking possibilities were used and the resulting $P$-value closest to the significance level was reported. The test of association (contingency) between FC ranges and location was performed with the Fisher Exact Test. Tests were two-sided with a significance level of 0.05 .

Simple linear regression analysis of LAZ (as the response variable) and FC was performed on the data from both groups. Because the rural data included a subset having FC values $>600 \mu \mathrm{g} / \mathrm{g}$ lacking precise measurement and the absence of these data would bias the regression results, Monte Carlo simulation was used to account for these data and estimate regression parameter values and confidence intervals. The simulated data were assigned FC values calculated with a function of the frequency distribution of the measured FC data and LAZ values were generated with random Gaussian variation from a line of linear association of LAZ and FC. The line and the magnitude of the variation were estimated from the frequency distribution and mean of the LAZ values of the 28 data, the median of the FC values $>600 \mu \mathrm{g} / \mathrm{g}$ and a slope determined from a series of preliminary simulations. One thousand sampling iterations were performed and in each case the 28 generated values were combined with the 77 accurately measured data and linear regression performed. Mean slope and intercept values and their 95\% confidence intervals were calculated from the results.

An estimate of the proportion of the mean negative LAZ for the rural group that was attributable to gut inflammation as indicated by the FC values for that group was calculated as the difference, expressed as a percentage of the mean negative LAZ, between the mean rural LAZ and the LAZ calculated from the urban (normal control) median FC value using the rural regression line.
Analyses were accomplished using Graphpad Prism (version 5.04, GraphPad Software, San Diego CA USA) and $\mathrm{R}$ (version 2.13, The R Project for Statistical Computing, www.r-project.org).

\section{Results}

The general characteristics and anthropometric data of the infants are shown in Table 1 . There were 144 infants, 105 from the rural area and 39 from the urban area, $75 \mathrm{M} / 69 \mathrm{~F}$. The mean age was $6.1 \pm 0.2 \mathrm{mo}$ (range: 5 mo $17 \mathrm{~d}$ to $6 \mathrm{mo} 30 \mathrm{~d}$. The mean values of LAZ, WAZ and WLZ for the rural infants were significantly lower than those for the urban infants $(P$-values $<0.0001,<0.0001$ and 0.0003 , respectively). The WLZ scores for males and females were significantly different for both the urban and rural infants $(P=0.022$ and 0.007 , respectively). All rural infants were breast fed; 20 of the urban infants were not receiving any breast milk at the time of the study and 50\% received some infant formula. Only a few Xichou infants received formula. All infants in both groups received some complementary foods by the age of four months, including rice, egg yolk and fruit.

The mean values of LAZ, WAZ and WLZ for all subjects with $\mathrm{FC}$ values $<350 \mu \mathrm{g} / \mathrm{g}$, a reported upper cut off during the first year of life [17], were $-0.0007,0.315$ and 0.517 respectively, compared to $-0.753,-0.427$ and 0.136 for subjects with $\mathrm{FC}$ values $>350 \mu \mathrm{g} / \mathrm{g}$. In each case the difference was significant $(P<0.0001,<0.0001$ and 0.021 respectively).

The median FC level was $281.5 \mu \mathrm{g} / \mathrm{g}$ (known range: $24.5 \sim 1267.9 \mu \mathrm{g} / \mathrm{g})$ in all infants. The median FC level was significantly higher in rural infants $(420.9 \mu \mathrm{g} / \mathrm{g}$, known range: $24.5-1268 \mu \mathrm{g} / \mathrm{g}$ ) than in urban infants $(140.1 \mu \mathrm{g} / \mathrm{g}$, known range 26.2-1258 $\mu \mathrm{g} / \mathrm{g})(P<0.0001)$. The median FC levels were not different for the 75 males $(243.8 \mu \mathrm{g} / \mathrm{g}$, known range: $26.2-1034 \mu \mathrm{g} / \mathrm{g})$ and 69 females (300.9 $\mu \mathrm{g} / \mathrm{g}$, known range: 24.5-1268 $\mu \mathrm{g} / \mathrm{g}$ ). Fifty-six percent and $10 \%$ of rural and urban measurements, respectively, were $>350 \mu \mathrm{g} / \mathrm{g}$. This association of FC range and location was significant $(P<0.0001)$.

Table 1 Characteristics of the study population ${ }^{1}$

\begin{tabular}{|c|c|c|c|c|c|c|}
\hline & \multicolumn{3}{|c|}{ Rural infants } & \multicolumn{3}{|c|}{ Urban infants } \\
\hline & $\begin{array}{c}\text { All } \\
(n=105)\end{array}$ & $\begin{array}{c}\text { Male } \\
(n=55)\end{array}$ & $\begin{array}{l}\text { Female } \\
(n=50)\end{array}$ & $\begin{array}{c}\text { All } \\
(n=39)\end{array}$ & $\begin{array}{c}\text { Male } \\
(n=20)\end{array}$ & $\begin{array}{l}\text { Female } \\
(n=19)\end{array}$ \\
\hline Age, mo & $6.0 \pm 0.2$ & $6.0 \pm 0.2$ & $6.1 \pm 0.2$ & $6.2 \pm 0.3$ & $6.3 \pm 0.3$ & $6.2 \pm 0.2$ \\
\hline Birth wt, kg & $3.1 \pm 0.4$ & $3.2 \pm 0.4$ & $3.0 \pm 0.4$ & $3.4 \pm 0.5$ & $3.5 \pm 0.6$ & $3.3 \pm 0.4$ \\
\hline Weight, kg & $7.4 \pm 0.9$ & $7.8 \pm 0.8$ & $7.0 \pm 0.8$ & $8.4 \pm 0.9$ & $8.9 \pm 0.7$ & $7.9 \pm 0.8$ \\
\hline Length, $\mathrm{cm}$ & $65.5 \pm 2.2$ & $66.1 \pm 2.1$ & $64.7 \pm 2.1$ & $67.8 \pm 2.3$ & $68.6 \pm 2.0$ & $67.0 \pm 2.3$ \\
\hline $\mathrm{LAZ}^{2}$ & $-0.6 \pm 0.9$ & $-0.7 \pm 0.9$ & $-0.5 \pm 0.9$ & $0.4 \pm 0.9$ & $0.4 \pm 0.9$ & $0.5 \pm 1.0$ \\
\hline$W^{\prime} Z^{3}$ & $-0.3 \pm 1.0$ & $-0.1 \pm 1.0$ & $-0.5 \pm 1.0$ & $0.8 \pm 0.8$ & $1.0 \pm 0.7$ & $0.6 \pm 0.8$ \\
\hline$\overline{W L Z^{4}}$ & $0.2 \pm 1.0$ & $0.4 \pm 0.9$ & $-0.1 \pm 1.0$ & $0.8 \pm 0.8$ & $1.1 \pm 0.7$ & $0.6 \pm 0.7$ \\
\hline
\end{tabular}

${ }^{1}$ Data are presented as mean \pm SD; ${ }^{2}$ Length-for-age Z-score; ${ }^{3}$ Weight-for-age Z-score; ${ }^{4}$ Weight-for-length Z-score. 
Simple linear regression of LAZ vs. FC for the urban infants showed that the relationship was not significant (Figure 1, $P=0.34$ ). Addition of an estimate for the single datum having an unknown value $>600 \mu \mathrm{g} / \mathrm{g}$ had negligible effect on these results. Regression analysis of the rural data using Monte Carlo simulation indicated that the relationship of LAZ and FC was significant $(P=0.014)$; the estimate for the slope was -0.00061 with a $95 \%$ confidence interval of -0.00013 to -0.00109 . The slope confidence interval indicated that LAZ and FC had a significant inverse relationship; more specifically, a $100 \mu \mathrm{g} / \mathrm{g}$ increase in FC was associated with a $0.06 \mathrm{de}-$ crease in LAZ. Examination of the residuals indicated no apparent departures from the regression assumptions. There was no correlation between birth weight and FC.

The proportion of the low mean LAZ for the rural group (-0.6) attributable to high FC was estimated to be $31 \%$ on average.

\section{Discussion}

It should be emphasized that this was an exploratory study included as a last minute addition to the baseline data for the parent study in Xichou followed by recognition of the potential interest in comparing with the upper-middle income urban children. Sample sizes were dictated by timelines rather than power testing. Statistics were complicated by some values for calprotectin in Xichou infants exceeding expectations and, therefore, the assay range established for the first batch. Hence, this should be regarded as a pilot observational study and our discussion viewed with this in mind.

In our model, the higher median FCC of the rural vs urban (control) infants accounted for one/third of the linear growth deficit. The results of this project suggest that the measurement of calprotectin concentrations in

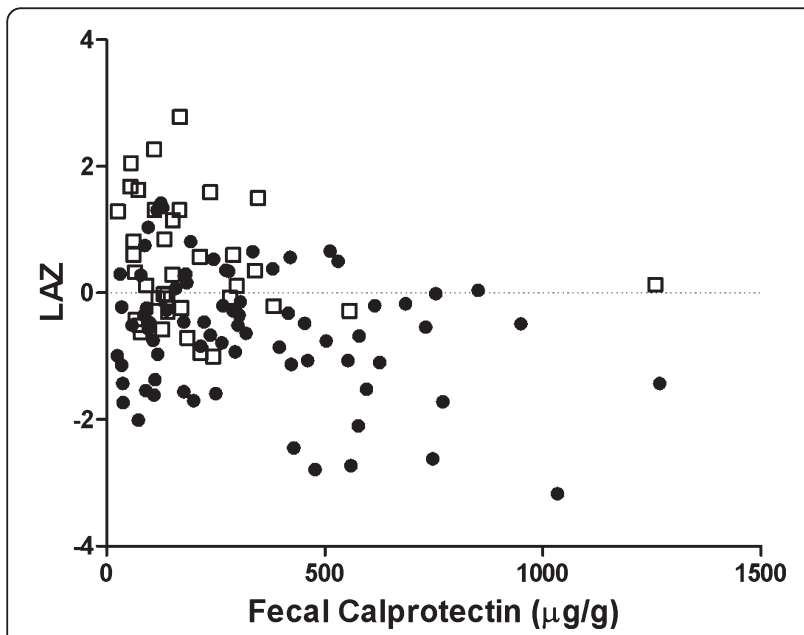

Figure 1 Length-for-age Z-scores as a function of fecal calprotectin. Symbols denote rural (•) and urban ( $\square$ ) infants. small random fecal samples provides a simple, noninvasive strategy for the detection of gut inflammation in apparently normal infants and a measure of the severity of this inflammation. It has been hypothesized that in these circumstances there is translocation of immunogenic luminal macromolecules across a compromised gut mucosa leading to stimulation of systemic immuno/ inflammatory processes and subsequent linear growth impairment [2]. The estimated contribution to linear growth faltering is less than that estimated for rural Gambian infants [2]. In contrast, FC levels were not elevated in urban children aged 0-12 y in urban Kampala [18] which suggests either a more favorable environment or a protective effect associated with increasing age. The median FC value of $140 \mu \mathrm{g} / \mathrm{g}$ for the urban infants was a little higher than that for the only other reported data for normal infants) at the age of 6 mo [19]. Our urban data, therefore, provided a quite conservative control for comparison with the rural infant data. It is noted that fecal CP levels were subsequently found to be modestly higher for age in hospitalized young children in Kampala who were HIV-infected, highly active anti-retroviralnaïve Ugandan children. Children with advanced disease had significantly higher FC levels and this also applied to older children with diarrhea [20].

Consistent with previous studies [19,21-26], the FC levels observed in healthy 6 mo infants in the current study were higher than in healthy adults and children. The high FC levels in infants may reflect the increased trans-epithelial migration of neutrophil granulocytes and/or macrophages into the intestinal lumen, which could be due to the greater intestinal permeability of young infants and may reflect an increase in leukocyte migration through the gut mucosa, as part of the development of the gut-associated lymphoid tissue which is particular to this period of life [27].

As ages of urban and rural infants were the same, agerelated differences did not account for the rural versus urban observed differences in FC. Breast feeding has in some, but not all, reports have been associated with higher FC levels in younger infants [24,27]. However, this difference does not account for the high $\mathrm{FC}$ value in the rural group at 6 months nor for the association between FC and linear growth in the rural group. Calprotectin levels are already used and documented extensively in the monitoring of the extent of inflammation of the gut in inflammatory bowel disease [28-30]. However, the application of this assay in assessing the integrity of the gut mucosa in infants with growth failure requires further studies prospectively designed to evaluate this as well as other recently proposed inflammatory markers that can be assayed in the feces. FC has the special advantage in infants and young children of not requiring venipuncture. The simplicity of collection and storage add further advantages. 


\section{Conclusion}

These results are consistent with the hypothesis that chronic sub-clinical intestinal inflammation is a greater problem in relatively poor rural infants in contrast to those living in a relatively affluent urban environment. Moreover, this apparent chronic low-grade inflammation is associated with impairment of linear growth.

\section{Abbreviations \\ FC: Fecal calprotectin; LAZ: Length-for-Age Z-score; WAZ: Weight-for-Age Z-score; WLZ: Weight-for-Length Z-score.}

\section{Competing interests}

None of the authors have any competing interests to disclose.

\section{Author contributions}

$\mathrm{KMH}, \mathrm{NFK}$, and XYS conceived of and designed the research project. XYS had oversight responsibility for the studies undertaken in China including the human studies in Yunnan province and the fecal calprotectin assays in Shanhgai. JRL and YQH were leaders in outcome assessment and data collection, processing. LVM assisted with the data analysis. JRL, KMH, LVM, XYS, NFK, and JEW prepared the manuscript and KMH, XYS and LVM had primary responsibility for final content. All authors have read and approved the final manuscript.

\section{Acknowledgements}

A special acknowledgement to Dr Jiang (head of maternal and child health services Xichou county, Yunnan province) and her staff without whose permission and very active support this study would not have been possible. The support included distribution of the intervention foods with the assistance of the directors of the ten hospitals in Xichou county and compliance monitoring.

This work was supported by the Thrasher Research Foundation 200708-29, National Nautral Science Foundation of China 81172686, Shanghai Key Laboratory of Pediatric Gastroenterology and Nutrition 11DZ2260500. Sponsors did not have a role in the design, collection, analyses or interpretation of data; in the writing of the manuscript; or in the decision to submit the manuscript for publication.

\section{Author details}

${ }^{1}$ Department of Child and Adolescent Health Care, MOE-Shanghai Key Laboratory of Children's Environmental Health, Xinhua Hospital, Shanghai Jiao Tong University, School of Medicine, Shanghai Key Laboratory of Pediatric Gastroenterology and Nutrition, Shanghai Institute for Pediatric Research, 1665 Kongjiang Road, Shanghai 200092, China. ${ }^{2}$ University of Colorado School of Medicine, Department of Pediatrics, Section of Nutrition, Box C225, Research Complex II, 12700 East 19th Avenue, Aurora, CO 80045, USA.

Received: 26 January 2012 Accepted: 16 August 2012

Published: 23 August 2012

\section{References}

1. Solomons NW, Mazariegos M, Brown KH, Klasing K: The underprivileged, developing country child: environmental contamination and growth failure revisited. Nutr Rev 1993, 51(11):327-332.

2. Campbell DI, Elia M, Lunn PG: Growth faltering in rural Gambian infants is associated with impaired small intestinal barrier function, leading to endotoxemia and systemic inflammation. J Nutr 2003, 133(5):1332-1338.

3. Panter-Brick C, Lunn PG, Goto R, Wright CM: Immunostimulation and growth faltering in UK infants. Am J Human Biol 2004, 16(5):581-587.

4. Campbell DI, McPhail G, Lunn PG, Elia M, Jeffries DJ: Intestinal inflammation measured by fecal neopterin in Gambian children with enteropathy: association with growth failure, Giardia lamblia, and intestinal permeability. J Pediatr Gastroenterol Nutr 2004, 39(2):153-157.

5. Lunn PG: The impact of infection and nutrition on gut function and growth in childhood. Proc Nutr Soc 2000, 59(1):147-154.
6. Goto R, Mascie-Taylor CG, Lunn PG: Impact of intestinal permeability, inflammation status and parasitic infections on infant growth faltering in rural Bangladesh. Brit J Nutr 2009, 101(10):1509-1516.

7. Panter-Brick C, Lunn PG, Langford RM, Maharjan M, Manandhar DS: Pathways leading to early growth faltering: an investigation into the importance of mucosal damage and immunostimulation in different socio-economic groups in Nepal. Brit J Nutr 2009, 101(4):558-567.

8. Dale I, Brandtzaeg P, Fagerhol MK, Scott H: Distribution of a new myelomonocytic antigen (L1) in human peripheral blood leukocytes. Immunofluorescence and immunoperoxidase staining features in comparison with lysozyme and lactoferrin. Am J Clin Pathol 1985, 84(1):24-34.

9. Roseth AG, Aadland E, Jahnsen J, Raknerud N: Assessment of disease activity in ulcerative colitis by faecal calprotectin, a novel granulocyte marker protein. Digestion 1997, 58(2):176-180.

10. Roseth $A G$, Schmidt PN, Fagerhol MK: Correlation between faecal excretion of indium-111-labelled granulocytes and calprotectin, a granulocyte marker protein, in patients with inflammatory bowel disease. Scand J Gastroenterol 1999, 34(1):50-54.

11. Gisbert JP, McNicholl AG, Gomollon F: Questions and answers on the role of fecal lactoferrin as a biological marker in inflammatory bowel disease. Inflamm Bowel Dis 2009, 15(11):1746-1754.

12. van Rheenen PF, Van de Vijver E, Fidler V: Faecal calprotectin for screening of patients with suspected inflammatory bowel disease: diagnostic meta-analysis. BMJ 2010, 341:C3369.

13. Canani RB, Terrin G, Rapacciuolo L, Miele E, Siani MC, Puzone C, Cosenza L, Staiano A, Troncone R: Faecal calprotectin as reliable non-invasive marker to assess the severity of mucosal inflammation in children with inflammatory bowel disease. Dig Liver Dis 2008, 40(7):547-553.

14. Berni Canani R, Rapacciuolo L, Romano MT, Tanturri De Horatio L, Terrin G, Manguso F, Cirillo P, Paparo F, Troncone R: Diagnostic value of faecal calprotectin in paediatric gastroenterology clinical practice. Digestive Liver Dis 2004, 36(7):467-470.

15. Hanevik K, Hausken T, Morken MH, Strand EA, Morch K, Coll P, Helgeland L, Langeland N: Persisting symptoms and duodenal inflammation related to Giardia duodenalis infection. J Infection 2007, 55(6):524-530.

16. Ton H, Brandsnes, Dale S, Holtlund J, Skuibina E, Schjonsby H, Johne B: Improved assay for fecal calprotectin. Clin Chim Acta 2000, 292(1-2):41-54.

17. Ezri J, Nydegger A: [Pediatrics. Fecal calprotectin in children: use and interpretation]. Rev Med Suisse 2011, 7(277):69-70.

18. Hestvik E, Tumwine JK, Tylleskar T, Grahnquist L, Ndeezi G, Kaddu-Mulindwa $\mathrm{DH}$, Aksnes L, Olafsdottir E: Faecal calprotectin concentrations in apparently healthy children aged 0-12 years in urban Kampala, Uganda: a community-based survey. BMC Pediatr 2011, 11:9.

19. Rugtveit J, Fagerhol MK: Age-dependent variations in fecal calprotectin concentrations in children. J Pediatr Gastroenterol Nutr 2002, 34(3):323-324. author reply 324-325.

20. Hestvik E, Olafsdottir E, Tylleskar T, Aksnes L, Kaddu-Mulindwa D, Ndeezi G, Tumwine JK, Grahnquist L: Faecal calprotectin in HIV-infected, HAART-naive Ugandan children. J Pediatr Gastroenterol Nutr 2012, 54(6):785-790.

21. Campeotto F, Butel MJ, Kalach N, Derrieux S, Aubert-Jacquin C, Barbot L, Francoual C, Dupont C, Kapel N: High faecal calprotectin concentrations in newborn infants. Arch Dis Child Fetal Neonatal Ed 2004, 89(4):F353-F355.

22. Dorosko SM, Mackenzie T, Connor Rl: Fecal calprotectin concentrations are higher in exclusively breastfed infants compared to those who are mixed-fed. Breastfeeding Med 2008, 3(2):117-119.

23. Olafsdottir E, Aksnes L, Fluge G, Berstad A: Faecal calprotectin levels in infants with infantile colic, healthy infants, children with inflammatory bowel disease, children with recurrent abdominal pain and healthy children. Acta Paediatr 2002, 91(1):45-50.

24. Savino F, Castagno E, Calabrese R, Viola S, Oggero R, Miniero R: High faecal calprotectin levels in healthy, exclusively breast-fed infants. Neonatology 2010, 97(4):299-304.

25. Nissen AC, van Gils CE, Menheere PP, Van den Neucker AM, van der Hoeven $M A$, Forget PP: Fecal calprotectin in healthy term and preterm infants. J Pediatr Gastroenterol Nutr 2004, 38(1):107-108.

26. Fagerberg UL, Loof L, Merzoug RD, Hansson LO, Finkel Y: Fecal calprotectin levels in healthy children studied with an improved assay. J Pediatr Gastroenterol Nutr 2003, 37(4):468-472. 
27. Kapel N, Campeotto F, Kalach N, Baldassare M, Butel MJ, Dupont C: Faecal calprotectin in term and preterm neonates. J Pediatr Gastroenterol Nutr 2010, 51(5):542-547.

28. Burri E, Beglinger C: Faecal calprotectin - a useful tool in the management of inflammatory bowel disease. Swiss Med Wkly 2012, 142:W13557.

29. van Rheenen PF: Role of fecal calprotectin testing to predict relapse in teenagers with inflammatory bowel disease who report full disease control. Inflamm Bowel Dis 2012, doi:10.1002/ibd.22896 [Epub ahead of print].

30. Fundaro C, Fantacci C, Ansuini V, Giorgio V, Filoni S, Barbaro F, Gasbarrini A, Rossi C: Fecal calprotectin concentration in children affected by SIBO. Eur Rev Med Pharmacol Sci 2011, 15(11):1328-1335.

doi:10.1186/1471-2431-12-129

Cite this article as: Liu et al.: Fecal calprotectin levels are higher in rural than in urban Chinese infants and negatively associated with growth. BMC Pediatrics 2012 12:129.

\section{Submit your next manuscript to BioMed Central and take full advantage of:}

- Convenient online submission

- Thorough peer review

- No space constraints or color figure charges

- Immediate publication on acceptance

- Inclusion in PubMed, CAS, Scopus and Google Scholar

- Research which is freely available for redistribution 